eCommons@AKU

April 2007

\title{
Modified MDRD equations outperform the original equations in Chinese patients with chronic kidney disease
}

Tazeen H. Jafar

Aga Khan University, tazeen.jafar@aku.edu

Follow this and additional works at: https://ecommons.aku.edu/pakistan_fhs_mc_med_nephrol Part of the Nephrology Commons

\section{Recommended Citation}

Jafar, T. H. (2007). Modified MDRD equations outperform the original equations in Chinese patients with chronic kidney disease. Nature Clinical Practice. Nephrology, 3(4), 186-187.

Available at: https://ecommons.aku.edu/pakistan_fhs_mc_med_nephrol/30 


\section{Modified MDRD equations outperform the original equations in Chinese patients with chronic kidney disease}

\begin{abstract}
Original article Ma Y-C et al. (2006) Modified glomerular filtration rate estimating equation for Chinese patients with chronic kidney disease. J Am Soc Nephrol 17: 2937-2944
\end{abstract}

\section{SYNOPSIS}

KEYWORDS Chinese patients, chronic kidney disease, glomerular filtration rate, MDRD, prediction equation

\section{BACKGROUND}

There is evidence that the MDRD (Modification of Diet in Renal Disease) equations underestimate glomerular filtration rate (GFR) in Chinese patients with mild renal insufficiency, and overestimate GFR in those with severe renal failure.

\section{OBJECTIVE}

To modify the full and abbreviated MDRD equations in order to improve their predictive power in Chinese patients with chronic kidney disease (CKD).

\section{DESIGN AND INTERVENTION}

Between June 2004 and September 2005, this study enrolled adults with CKD at 9 university hospitals in China. Exclusion criteria included edema, heart failure and need for renal replacement therapy. The reference GFR value was determined at each center from the plasma clearance of ${ }^{99 \mathrm{~m} T c-D T P A}$. Plasma creatinine levels were analyzed centrally; a random selection of plasma samples was also analyzed in the Cleveland Clinic Laboratory to allow calibration of the values to the MDRD equations. Using data from a randomly selected subset of patients, the investigators employed linear regression to produce modified versions of the full and abbreviated MDRD equations that incorporated a factor accounting for Chinese race. A further two equations were constructed by refitting the MDRD equations to the same subset of patients using raw plasma creatinine values (i.e. without calibration to the MDRD laboratory). The predictive performance of the new equations in the remaining patients was compared with that of the original MDRD equations. See Supplementary Information online for the full equations.

\section{OUTCOME MEASURES}

The bias, precision and accuracy of the modified equations were assessed.

\section{RESULTS}

Of the 684 patients included in the study (mean age 49.98 years; $48.5 \%$ female), a random sample of 454 provided the data for modification of the equations. The remaining 230 were used to validate the modified equations. The four modified MDRD equations showed significantly higher $15 \%$ accuracy than the two original equations (46.9-50.4\% vs $32.6 \%$ for the full MDRD equation and $30.0 \%$ for the abbreviated equation; $P<0.05$ in all cases). The modified equations had slightly better precision (narrower limits of agreement) than the original equations, ranging from 53.2 to $57.5 \mathrm{ml} / \mathrm{min} / 1.73 \mathrm{~m}^{2}$ (compared with $57.6 \mathrm{ml} / \mathrm{min} / 1.73 \mathrm{~m}^{2}$ for the full MDRD equation and $60.7 \mathrm{ml} / \mathrm{min} / 1.73 \mathrm{~m}^{2}$ for the abbreviated equation). Bias of the modified equations was much lower than that of the original equations (543-686 vs $2,134-2,175)$. When the modified equations were analyzed in each stage of CKD separately, they had significantly greater $15 \%$ accuracy than the original equations in stages 1 and $2(P<0.05$ for all). In stage $1 C K D$, fewer patients were misclassified as CKD stage 2 by the new equations than by the original equations $(45.2-54.8 \%$ vs $71.4-73.8 \%)$. In stage 2 CKD, fewer patients were misclassified by the new equations as stage $3(30.0-31.7 \%$ vs 60.0-68.3\%). A final four modified equations were generated using the entire dataset (see Supplementary Information online).

\section{CONCLUSION}

The modified MDRD equations provide better estimates of GFR in Chinese patients with CKD than do the original equations. 


\section{COMMENTARY}

\section{Tazeen H Jafar}

CKD is a major public health problem worldwide, with adverse outcomes including loss of kidney function, sometimes leading to kidney failure, and cardiovascular disease. Unfortunately, CKD is underdiagnosed and undertreated.

Early detection of CKD can be achieved using markers of kidney damage or more-sensitive measures of kidney function, such as estimated GFR. Several formulae, including the MDRD equation, have been developed to estimate GFR based on serum creatinine concentration, age, sex, and body size. Many organizations recommend using these equations to detect, evaluate and manage CKD. ${ }^{1}$

In general, the MDRD equation demonstrates minimal bias and reasonable precision and accuracy in white patients with CKD and mean GFR $<60 \mathrm{ml} / \mathrm{min} / 1.73 \mathrm{~m}^{2}$. More than $90 \%$ of estimates fall within $30 \%$ of the measured value. ${ }^{2}$ The picture is less clear in predominantly non-CKD populations (such as kidney donors or patients with diabetes and high GFR), and in nonwhite populations. The relationships between the variables used in GFR estimation equations and the non-GFR determinants of serum creatinine level (e.g. creatinine generation, secretion and extrarenal elimination) can vary according to health status and lifestyle factors (e.g. diet). ${ }^{3}$ This variation might lead to increased errors when estimating GFR in certain ethnic groups that differ from the population in whom the equations were originally developed (i.e. whites and African Americans in the US). ${ }^{4}$ Consistent with this notion, MDRD estimates correlate poorly with measured GFR in Chinese patients with CKD. ${ }^{5}$

The study by Ma et al. examined the performance of the MDRD equation in Chinese patients with various stages of $C K D$, and compared it with that of modified equations developed specifically for the study population. The modified equations were generated by adding a term for Chinese race to the MDRD equation, or by refitting the MDRD equation to the study population using the same variables as were originally used. The overall performance of the modified equations was superior to that of the original equations, with significant improvements in bias, precision, and accuracy.

The MDRD equation performed poorly in CKD stages 1 and 2, with about $60-70 \%$ misclassification. It is important to note that almost half of the subjects with CKD stages 1 or 2 were still misclassified by the modified equations. Further, in CKD stages 3-5, there were no significant differences in performance between the original and modified equations. Unfortunately, the investigators did not report the performance of the Cockcroft-Gault equation, nor did they test the addition of variables to the MDRD equation that were not included in the original equation, but could potentially improve its performance in Chinese patients. Moreover, the study was limited to patients with $\mathrm{CKD}$, thus restricting its relevance to those with high GFR, and to non-CKD populations at high risk for this condition (e.g. patients with hypertension or diabetes). Nevertheless, this important study highlights the challenges of searching for more accurate estimates of kidney function in Asian populations.

The practicing clinician should know that although the modified GFR equations seem to have some advantage over the original MDRD equations in Chinese patients, both the original and modified equations would generate high numbers of false-positives and false-negatives in non-CKD populations, and in individuals with CKD stages 1-2. Confirmation of the estimate with measurement of 24 -h creatinine clearance will still be required in these situations.

Supplementary information is available on the Nature Clinical Practice Nephrology website.

\section{References}

1 National Kidney Foundation (2002) K/DOQI clinical practice guidelines for chronic kidney disease: evaluation, classification, and stratification. $A m \mathrm{~J}$ Kidney Dis 39 (Suppl 1): S1-S266

2 Levey AS et al. (1999) A more accurate method to estimate glomerular filtration rate from serum creatinine: a new prediction equation. Modification of Diet in Renal Disease Study Group. Ann Intern Med 130: $461-470$

3 Stevens LA et al. (2006) Assessing kidney functionmeasured and estimated glomerular filtration rate. N Engl J Med 354: 2473-2483

4 Jafar TH et al. (2005) Serum creatinine as marker of kidney function in South Asians: a study of reduced GFR in adults in Pakistan. J Am Soc Nephrol 16: 1413-1419

5 Zuo Let al. (2005) Application of GFR-estimating equations in Chinese patients with chronic kidney disease. Am J Kidney Dis 45: 463-472

TH Jafar is Head of the Section of Nephrology, Director of the Clinical Epidemiology Unit and Associate Professor in the Departments of Medicine and Community Health Sciences at the Aga Khan University, Karachi, Pakistan.
Acknowledgments

The synopsis was written by Chloë Harman, Associate Editor, Nature Clinical Practice.

\section{Competing interests}

The author declared she has no competing interests.

\section{Correspondence}

Department of Medicine Aga Khan University

Stadium Road

PO Box 3500

Karachi 74800

Pakistan

tazeen.jafar@aku.edu

$\overline{\text { Received } 26 \text { November } 2006}$ Accepted 10 January 2007 Published online 13 February 2007

www.nature.com/clinicalpractice doi:10.1038/ncpneph0428
PRACTICE POINT A modified form of the MDRD equation devised specifically for Chinese patients might be preferable to the original equation for estimation of GFR in relevant Asian populations if its superiority is confirmed in subsequent studies 